\title{
SOME REMARKS ON TABOO PROBABILITIES
}

BY

\author{
K. L. Chung ${ }^{1}$
}

Consider a discrete-parameter homogeneous Markov chain $\left\{x_{n}, n \geqq 0\right\}$ with state space I and one-step transition matrix $\left(\left(p_{i j}\right)\right), i, j \in \mathbf{I}$. For any subset $H$ of $\mathbf{I}$ we define the taboo probability

and set

$$
{ }_{H} p_{i j}^{(n)}=\mathbf{P}\left\{x_{n}=j ; x_{\nu} \notin H, 0<\nu<n \mid x_{0}=i\right\}, \quad n \geqq 1 \text {, }
$$

$$
{ }_{H} p_{i j}^{*}=\sum_{n=1 H}^{\infty} p_{i j}^{(n)} \text {. }
$$

When $H=\{k\}$ we write ${ }_{k} p_{i j}^{(n)}$ for ${ }_{H} p_{i j}^{(n)}$. Furthermore we write $f_{i j}^{(n)}$ for ${ }_{j} p_{i j}^{(n)}$ and $e_{i j}^{(n)}$ for ${ }_{i} p_{i j}^{(n)}$. Thus,

$$
f_{i j}^{*}=\sum_{n=1}^{\infty} f_{i j}^{(n)}, \quad e_{i j}^{*}=\sum_{n=1}^{\infty} e_{i j}^{(n)} .
$$

The quantity $f_{i j}^{*}$ is familiar, the quantity $e_{i j}^{*}$ has been studied in [1] under the notation $e_{i j}$. We set also

and ${ }_{H} p_{i j}^{(0)}=\delta_{i j}$.

$$
m_{i j}=\sum_{n=1}^{\infty} n f_{i j}^{(n)}
$$

In this paper a "well known" statement means one which can be found in [1], particularly §I.9 there which treats taboo probabilities. What follows may be regarded as some interesting corollaries of well-known results which seem worth stating. They are engendered by a generalization (Proposition 7) of a recent result of Spitzer [3]. This will be placed where it properly belongs, and the proof will be strictly elementary. In doing so we shall define a new binary relation between the states of a Markov chain.

Recall that two states $i$ and $j$ belong to the same recurrent class if and only if $f_{i j}^{*}=f_{j i}^{*}=1$, or alternatively $f_{i i}^{*}=1$ and $f_{i j}^{*}>0 . \quad$ A subset $C$ of $\mathbf{I}$ is said to form an equitable class if and only if for every $i$ and $j$ in $C$ we have

$$
e_{i j}^{*}=1 \text {. }
$$

A well-known example of an equitable class is the following: $x_{n}$ is the sum of $n$ independent and identically distributed random variables with mean zero, or more generally, $x_{n}$ is a recurrent Markov chain with stationary and independent increments.

We have the following characterization.

Proposition 1. An equitable class $C$ is recurrent. A recurrent class is equitable if and only if for each $i$ in $C$, we have

$$
\sum_{j \in C} p_{j i}=1
$$

namely when $\left(\left(p_{i j}\right)\right)$ restricted to $C$ is doubly stochastic.

Received October 6, 1960.

${ }_{1}^{1}$ This work is supported in part by the Office of Scientific Research of the United States Air Force. 
Proof. The first assertion is trivial since $f_{i i}^{*}=e_{i i}^{*}=1$, and $e_{i j}^{*}=1$ implies $f_{i j}^{*}>0$. Hence the second criterion for recurrence cited above applies. Next, by a well-known theorem of Derman, the following system of equations

$$
u_{i}=\sum_{j \epsilon C} u_{j} p_{j i}, \quad i \epsilon C,
$$

has the unique nonnegative solutions $\left\{u_{j}, j \in C\right\}$ given by

$$
u_{j}=c_{i} e_{i j}^{*}
$$

for an arbitrary $i \epsilon C$ and an arbitrary $c_{i} \geqq 0$. This and the fact that $e_{i i}^{*}=1$ establish the second assertion of the proposition.

Proposition 2. An equitable class $C$ is positive-recurrent if and only if $C$ is a finite set and

$$
m_{i i}=c
$$

for each $i \epsilon C$, where $c$ is the cardinal of $C$.

Proof. It is well known that the recurrent class $C$ is positive or null according as $\sum_{j \epsilon C} e_{i j}^{*}<\infty$ or $=\infty$. In the former case it is also well known that

$$
e_{i j}^{*}=m_{j j} / m_{i i} \quad \text { and } \quad \sum_{i \epsilon C}\left(1 / m_{i i}\right)=1 \text {. }
$$

The proposition follows from these facts.

Proposition 3. For a class $C$ to be equitable it is necessary and sufficient that there exists an $i$ in $C$ such that we have $e_{i j}^{*}=1$ for each $j$ in $C$.

Proof. Necessity is just a part of the definition of equitability. To prove sufficiency we observe that $C$ must be recurrent since $i$ is. Now it is well known that in a recurrent class we have

$$
e_{i j}^{*} e_{j k}^{*}=e_{i k}^{*} .
$$

It follows that $e_{j k}^{*}=1$ for every $j$ and $k$, proving that $C$ is equitable.

Two distinct states $i$ and $j$ are said to be an equitable pair if and only if

$$
e_{i j}^{*}=e_{j i}^{*}=f_{i j}^{*}=f_{j i}^{*}=1 \text {. }
$$

It is important to note that an equitable pair of states does not necessarily belong to an equitable class, since the latter need not exist.

Proposition 4. If $i$ and $j$ are an equitable pair, then they belong to the same recurrent class $C$, and $e_{i k}^{*}=e_{j k}^{*}$ for each $k$ in $C$. Furthermore we have

$$
{ }_{j} p_{i i}^{*}={ }_{i} p_{j j}^{*}, \quad{ }_{i} f_{i j}^{*}={ }_{j} f_{j i}^{*},
$$

where ${ }_{i} f_{i j}^{*}={ }_{H} p_{i j}^{*}, f_{j i}^{*}={ }_{H} p_{j i}^{*}$ with $H=\{i, j\}$.

Proof. The first assertion follows from the first criterion of recurrence cited before; the second from (4). The rest follows from the well-known 
relations:

$$
\frac{f_{i j}^{*}}{e_{i j}^{*}}=\frac{1+{ }_{j} p_{i i}^{*}}{1+{ }_{i} p_{j j}^{*}}=\frac{f_{i j j}^{*} f_{j i}^{*}}{f_{j i}^{*} f_{i j}^{*}}\left(=\lim _{n \rightarrow \infty} \frac{\sum_{\nu=1}^{n} p_{i i}^{(\nu)}}{\sum_{\nu=1}^{n} p_{j j}^{(\nu)}}\right) .
$$

(The one in the parenthesis is not needed to prove the others, but is the heart of the matter.)

The next proposition, in some form, has been mentioned to me by several persons, including Hoeffding, Orey, and C. J. Stone.

Proposirion 5. Whatever the states $i$ and $j$, we have

$$
0 \leqq \sum_{n=0}^{N}\left[p_{i i}^{(n)}-p_{j i}^{(n)}\right] \leqq \sum_{n=0}^{N} p_{i i}^{(n)}, \quad N \geqq 0 .
$$

If $e_{i j}^{*} \leqq 1$ and $e_{j i}^{*} \leqq 1$, then

$$
0 \leqq \sum_{n=0}^{N}\left[p_{j j}^{(n)}-p_{j i}^{(n)}\right] \leqq \sum_{n=0}^{N} p_{j j}^{(n)},
$$$$
N \geqq 0 \text {. }
$$

Remark. The "silent" condition for $(5)$ is of course $f_{i j}^{*} \leqq 1$ and $f_{j i}^{*} \leqq 1$. Observe also that if $i$ and $j$ are recurrent, and $e_{i j}^{*} \leqq 1, e_{j i}^{*} \leqq 1$, then $i$ and $j$ are in fact an equitable pair since $1=e_{i i}^{*}=e_{i j}^{*} e_{j i}^{*}$.

Proof. We prove (6) only, since (5) is similar and will not be used. We may suppose that $i \neq j$ in (6). By a well-known formula,

consequently

$$
p_{j j}^{(n)}={ }_{i} p_{j j}^{(n)}+\sum_{\nu=1}^{n} p_{j i}^{(\nu)} e_{i j}^{(n-\nu)}
$$

$$
\begin{aligned}
\sum_{n=0}^{N} p_{j j}^{(n)}=\sum_{n=0}^{N} p_{j j}^{(n)}+\sum_{\nu=1}^{N} p_{j i}^{(\nu)} & \sum_{n=1}^{N-\nu} e_{i j}^{(n)} \\
& \leqq \sum_{n=0}^{N} p_{j j}^{(n)}+\sum_{\nu=1}^{N} p_{j i}^{(\nu)},
\end{aligned}
$$

since $\sum_{n=1}^{N-\nu} e_{i j}^{(n)} \leqq e_{i j}^{*} \leqq 1$. On the other hand, we have by another wellknown formula:

$$
\begin{aligned}
p_{j i}^{(n)} & =\sum_{\nu=0}^{n} p_{j j}^{(\nu)} e_{j i}^{(n-\nu)} \\
\sum_{n=0}^{N} p_{j i}^{(n)} & =\sum_{\nu=0}^{N} p_{j j}^{(\nu)} \sum_{n=0}^{N-\nu} e_{j i}^{(n)} \leqq \sum_{\nu=0}^{N} p_{j j}^{(\nu)}
\end{aligned}
$$

since $\sum_{n=0}^{N=\nu} e_{j i}^{(n)} \leqq e_{j i}^{*} \leqq 1$. These inequalities establish (6).

The next proposition has nothing to do with probability; it is stated here without (trivial) proof for the sake of explicitness.

Proposition 6. If $\sum_{\nu}\left|a_{\nu}\right|<\infty,\left|b_{n}\right| \leqq A<\infty$ for all $n$, and $\lim _{n \rightarrow \infty}\left(b_{n}-b_{n-1}\right)=0$, then

$$
\lim _{n \rightarrow \infty} \sum_{\nu=1}^{n}\left|a_{\nu}\right|\left|b_{n}-b_{n-\nu}\right|=0 .
$$

Propositon 7. If $i$ and $j$ are an equitable pair and belong to either a nullrecurrent class or an aperiodic positive-recurrent class, then we have

$$
\sum_{n=0}^{\infty}\left\{p_{i i}^{(n)}+p_{j j}^{(n)}-p_{i j}^{(n)}-p_{j i}^{(n)}\right\}=1+{ }_{j} p_{i i}^{*} .
$$

Remark 1. It is well known that $1+{ }_{j} p_{i i}^{*}=\left({ }_{j} f_{j i}^{*}\right)^{-1}<\infty$ in a recurrent 
class. Since the left member of (7) is symmetric in $i$ and $j,(7)$ implies a partial proof of the second sentence in Proposition 4.

Remark 2. The proposition is not true if $i$ and $j$ belong to a periodic, positive-recurrent class; let $p_{i j}=1=p_{j i}$.

Proof. Write the trivial equations:

$$
\begin{aligned}
& \sum_{n=1}^{N} p_{j i}^{(n)}=\sum_{\nu=1}^{N} f_{i j}^{(\nu)} \sum_{n=1}^{N} p_{j i}^{(n)}+\sum_{\nu=N+1}^{\infty} f_{i j}^{(\nu)} \sum_{n=1}^{N} p_{j i}^{(n)}, \\
& \sum_{n=0}^{N} p_{j j}^{(n)}=\sum_{\nu=1}^{N} f_{i j}^{(\nu)} \sum_{n=0}^{N} p_{j j}^{(n)}+\sum_{\nu=N+1}^{\infty} f_{i j}^{(\nu)} \sum_{n=0}^{N} p_{j j}^{(n)} .
\end{aligned}
$$

By well-known formulas, we have

$$
\begin{aligned}
& \sum_{n=1}^{N} p_{i j}^{(n)}=\sum_{\nu=1}^{N} f_{i j}^{(\nu)} \sum_{n=0}^{N-\nu} p_{j j}^{(n)}, \\
& \sum_{n=0}^{N} p_{i i}^{(n)}=\sum_{\nu=1}^{N} f_{i j}^{(\nu)} \sum_{n=1}^{N-\nu} p_{j i}^{(n)}+\sum_{n=0}^{N} p_{i i}^{(n)} .
\end{aligned}
$$

Combining these four equations we obtain

$$
\begin{aligned}
\sum_{n=0}^{N}\left\{p_{i i}^{(n)}+p_{j j}^{(n)}-p_{i j}^{(n)}\right. & \left.-p_{j i}^{(n)}\right\}=\sum_{\nu=1}^{N} f_{i j}^{(\nu)} \sum_{n=N-\nu+1}^{N}\left[p_{j j}^{(n)}-p_{j i}^{(n)}\right] \\
& +\sum_{\nu=N+1}^{\infty} f_{i j}^{(\nu)} \sum_{n=0}^{N}\left[p_{j j}^{(n)}-p_{j i}^{(n)}\right]+\sum_{n=0}^{N} p_{i i}^{(n)} .
\end{aligned}
$$

As $N \rightarrow \infty$, the third term converges to $1+{ }_{j} p_{i i}^{*}$. The second term converges to zero by virtue of $(6)$, since ${ }_{i} p_{j j}^{*}<\infty$. For the first term we apply Proposition 6 with $a_{\nu}=f_{i j}^{(\nu)}$ and $b_{n}=\sum_{\nu=0}^{n}\left[p_{j j}^{(\nu)}-p_{j i}^{(\nu)}\right.$. The first condition in Proposition 6 is clearly satisfied, the second by virtue of (6). If $i$ and $j$ are in a null-recurrent class we have

$$
\lim _{n \rightarrow \infty}\left(b_{n}-b_{n-1}\right)=\lim _{n \rightarrow \infty}\left(p_{j j}^{(n)}-p_{j i}^{(n)}\right)=0-0=0 .
$$

If they are in an aperiodic, positive-recurrent class, the above limit is equal to

$$
1 / m_{j j}-1 / m_{i i}=0
$$

by Proposition 3. Hence in either case the third condition of Proposition 6 is also satisfied, and so the first term above converges to zero. Proposition 7 is proved.

When $x_{n}$ is the sum of $n$ independent and identically distributed integervalued random variables with mean zero, Proposition 7 reduces to Theorem 1 in Spitzer [3]. His Theorem 2 can be proved in a similar way.

The following question is open, even in Spitzer's case: Is the series $\sum_{n}\left\{p_{i i}^{(n)}-p_{j i}^{(n)}\right\}$ convergent in a null-recurrent, equitable class? The corresponding problem for a positive-recurrent class has been considered in [1]. As a particular case in Theorem I. 11.4 there, ${ }^{2}$ we have, if the class is aperiodic,

$$
\sum_{n=0}^{\infty}\left\{p_{i i}^{(n)}-p_{j i}^{(n)}\right\}=m_{j i} / m_{i i}
$$

${ }^{2}$ I take this opportunity to correct a foolish slip in [1] about this theorem. If the class has period $d$, the proof there yields the convergence of $\sum_{\nu=1}^{n d+r}\left\{p_{i k}^{(\nu)}-p_{j k}^{(\nu)}\right\}$ as $n \rightarrow \infty$, for each fixed $r$. The theorem as stated is correct when $d=1$; the corollary should be deleted. 
If the class is also equitable, then it follows that the left member of (7) is equal to

$$
m_{j i} / m_{i i}+m_{i j} / m_{j j}=\left(m_{j i}+m_{i j}\right) / m_{i i}
$$

by (3). The last-written fraction is well known to be equal to $1+{ }_{j} p_{i i}^{*}$, checking our previous result.

In a previous paper [2] I mentioned the problem of investigating the convergence of $\sum_{n}\left\{p_{i k}^{(n)}-p_{j k}^{(n)}\right\}$ in a null-recurrent class. Mr. C. J. Stone has recently informed me that this series need not converge. In this connection it may be worthwhile to record the following dual formulas. If $f_{i j}^{*}=1$, then for each $k$,

$$
\sum_{\nu=1}^{n}\left\{p_{i k}^{(\nu)}-p_{j k}^{(\nu)}\right\}={ }_{j} p_{i k}^{*}-\sum_{l \in \mathbf{I}{ }_{j}} p_{i l}^{*} p_{l k}^{(n)}
$$

if $e_{i j}^{*}=1$, then for each $k$,

$$
\sum_{\nu=1}^{n}\left\{p_{k i}^{(\nu)}-p_{k j}^{(\nu)}\right\}=\sum_{l \in \mathbf{I}} p_{k l}^{(n)}{ }_{i} p_{l j}^{*}-{ }_{i} p_{k j}^{*} .
$$

The first formula is in [1]; the second is proved dually.

Addendum. I am indebted to Hoeffding and Snell (independently) for the following extension of Proposition 7.

First, in formula (6), let $i$ and $j$ now belong to a recurrent class, but discard the assumption that $e_{i j}^{*} \leqq 1, e_{j i}^{*} \leqq 1$. Then without changing the proof there but noticing that $e_{i j}^{*} e_{j i}^{*}=1$, we obtain in lieu of $(6)$ :

$$
0 \leqq \sum_{n=0}^{N}\left[p_{j j}^{(n)}-e_{i j}^{*} p_{j i}^{(n)}\right] \leqq \sum_{n=0}^{N} p_{j j}^{(n)}, \quad N \geqq 0 .
$$

Now let $i$ and $j$ be distinct states belonging to a null-recurrent class. Multiplying (8) and (11) through by $e_{i j}^{*}$, forming the following combination, and proceeding as before, we obtain in lieu of $(7)$ :

$$
\sum_{n=0}^{\infty}\left\{e_{i j}^{*}\left(p_{i i}^{(n)}-p_{j i}^{(n)}\right)+p_{j j}^{(n)}-p_{i j}^{(n)}\right\}=e_{i j}^{*}\left(1+{ }_{j} p_{i i}^{*}\right)=1+{ }_{i} p_{j j}^{*} .
$$

Formula (13) is thus valid in any null-recurrent class and reduces to (7) when the class is also equitable.

\section{REFERENCES}

1. K. L. Chung, Markov chains with stationary probabilities, Berlin, Springer-Verlag, 1960.

2. - - Contributions to the theory of Markov chains, J. Res. Nat. Bur. Standards, vol. 50 (1953), pp. 203-208.

3. F. Spitzer, Some properties of recurrent random walk, Illinois J. Math., vol. 5 (1961), pp. 234-245.

\section{Syracuse University}

SYracuse, New YorK 